\title{
UNE RÉACTION : PENSER LA SORTIE DE L'IMAGINAIRE DE LA CROISSANCE AU TRAVERS DES INITIATIVES LOCALES DE TRANSITION ?
}

\section{Ludivine Damay, Anne Guisset}

Université Saint-Louis - Bruxelles | «Revue interdisciplinaire d'études juridiques » 2016/2 Volume 77 | pages 131 à 135

ISSN $0770-2310$

Article disponible en ligne à l'adresse :

https://www.cairn.info/revue-interdisciplinaire-d-etudesjuridiques-2016-2-page-131.htm

\section{(cc) BY-NC}


R.I.E.J., 2016.77

\title{
Une réaction : Penser la sortie de l'imaginaire de la croissance au travers des initiatives locales de transition?
}

\author{
Ludivine DAMAY \\ Professeure en sociologie à l'Université libre de Bruxelles, \\ Faculté d'Architecture La Cambre Horta \\ Professeure invitée à l'Université Saint-Louis - Bruxelles
}

Anne GUISSET

Doctorante en science politique au CReSPo, Université Saint-Louis - Bruxelles Boursière FRESH, F.R.S. - FNRS

\section{Introduction}

Le texte d'Olivier De Schutter propose deux métaphores pertinentes, la cage et le labyrinthe, pour évoquer d'une part l'enserrement de l'individu et de la société dans la mystique de la croissance et d'autre part l'erreur qui consisterait à penser qu'il n'y a qu'une voie possible pour s'en évader. L'argumentation, très convaincante et passionnante, convoque avec àpropos différents auteurs sur l'esprit du capitalisme, la manière dont il colonise nos pensées, la société de concurrence généralisée, etc. Nous proposons néanmoins une discussion de ce texte en deux temps. Dans une première partie, nous revenons sur quelques aspects plus théoriques, en nous appuyant sur certaines approches sociologiques, afin d'éclairer la manière dont se forme l'imaginaire capitaliste et dont on peut en sortir pour construire une société alternative. Dans un second temps, nous utilisons les premières analyses issues d'une recherche en cours sur les initiatives locales de transition dans la ville de Gembloux ${ }^{1}$ pour approfondir la question des motivations individuelles envers l'engagement dans ces mouvements. Si « l'utopie quotidienne » existe bien, comme l'affirme Olivier De Schutter, estelle forcément pour tous les acteurs qui s'y engagent porteuse d'une volonté de changement social? Le rapport au politique des acteurs engagés permet-il une diffusion de l'innovation sociale vers les autorités publiques ?

\section{Volonté individuelle et changement d'imaginaire}

D'abord, nous voudrions clarifier le fait que l'esprit du capitalisme n'est pas uniquement le fruit d'une transformation volontaire des mentalités individuelles. II est difficile d'affirmer que la « cage » a été produite par les

\footnotetext{
${ }^{1}$ Gembloux est une ville de 25.000 habitants située dans la province de Namur en Belgique.
} 
individus sans évoquer les conditions sociales de son apparition qui influent aussi de manière dynamique sur la transformation des structures psychiques des individus (pensons au développement des villes, à la division du travail, à l'existence d'un travail «libre », etc.). Pour revenir, comme Olivier De Schutter, à Max Weber, nous souhaitons insister sur le caractère non intentionnel de ce changement social selon lui: ce n'était pas l'intention des promoteurs de la Réforme, ni l'intention des protestants, que de développer le capitalisme. La Doctrine de la prédestination impliquait pour l'adepte de rechercher ici-bas les signes de son élection, signes visibles dans ses capacités à entreprendre, à réussir, mais aussi à réinvestir, en ne dilapidant pas son capital, en pratiquant «l'ascèse intramondaine ". Plutôt que de consommer, il s'agissait ainsi d'avoir une vie simple et sobre. "Le puritain voulait être besogneux et nous sommes forcés de l'être ${ }^{2}$, dit Weber. Si la Réforme protestante a accompagné les transformations subjectives nécessaires au développement du capitalisme, la relation entre les deux n'est donc pas simple, ni n'explique, par exemple, l'accumulation de biens de consommation. Peter Berger utilise d'ailleurs cet ouvrage de Weber pour relever l'« image frappante des paradoxes de l'action humaine ", l'histoire ne résultant pas uniquement des efforts délibérés des individus pour atteindre un but ${ }^{3}$.

Le texte d'Olivier De Schutter fait également référence à un « manque d'imagination dont souffre l'individu plongé dans le capitalisme ", celui-ci ne pouvant concevoir sa vie en dehors de ce modèle, bien aidé en cela par un " ensemble de dispositifs à l'échelle sociétale " qui renforcent ces dispositions. Cette position évoque évidemment la notion d'imaginaire, que l'on retrouve chez Cornelius Castoriadis mais aussi chez Serge Latouche, un économiste partisan de la décroissance. Ce dernier auteur pose une hypothèse semblable à celle d'Olivier De Schutter : "Si la croissance et le développement sont des croyances, donc des significations imaginaires sociales, comme le progrès et l'ensemble des catégories fondatrices de l'économie, pour en sortir, les abolir et les dépasser, il faut changer d'imaginaire $"{ }^{4}$. Ce qui pose sans doute davantage de questions, c'est la manière d'en changer: les individus peuvent-ils le décider? Pour Castoriadis, "le social structure l'individu de part en part, ce qui pose la question de savoir comment il peut devenir autonome ${ }^{5}$ et donc, pour le

${ }^{2}$ M. WeBER, L'éthique protestante et l'esprit du capitalisme, Paris, Pocket, 1990, p. 224.

${ }_{3}^{3}$ P. L. BeRger, Invitation à la sociologie, Paris, La Découverte, 2006, p. 73 (1963).

4 S. LATOUChe, Décoloniser l'imaginaire, in Décroissance. Vocabulaire pour une nouvelle ère, G. d'Alisa, F. Demaria, G. Kallis (dir.), Neuvy-en-Champagne, Le passager clandestin, 2015, p. 248.

${ }^{5}$ Ph. CAUMieres, Castoriadis : le projet d'autonomie, Paris, Michalon, 2007, p. 109. 
propos de notre contribution, comment il pourrait se déprendre d'un imaginaire capitaliste qui prédomine. "Un individu seul, ou une organisation, ne peut, au mieux, que préparer, critiquer, inciter, esquisser des orientations possibles ${ }^{6}$, affirme Castoriadis. Ce sont davantage des moments révolutionnaires, au cœur desquels se produisent des bouleversements des rapports sociaux qui permettraient ce changement. Latouche insiste également sur le fait que ce changement d'imaginaire est « très difficile, parce qu'on ne décide pas de changer son imaginaire et encore moins celui des autres, surtout s'ils sont accros à la croissance. " ${ }^{7}$. Cet imaginaire est tellement ancré, intériorisé, reproduit sans cesse dans les dispositifs de la vie sociale qu'il paraît difficile à faire évoluer. Pour Latouche, la décolonisation de l'imaginaire pourrait s'enclencher, avec comme point de départ de la contre-offensive, la lutte contre l'agression publicitaire. Un acte qui aurait des impacts sur tous et qui serait suffisamment radical pour enclencher un mouvement collectif.

La position d'Olivier De Schutter à propos des voies de la décolonisation possible renvoie quant à elle à plusieurs pistes, sans passer par le radicalisme d'un mouvement d'ensemble et qui procéderait d'un seul coup, proposant, si l'on peut dire, une stratégie de la contagion: développement des innovations sociales locales; participation des acteurs à la gouvernance; soutien des niveaux de gouvernances aux innovations; apprentissage collectif et réflexivité de la société. Dans la deuxième partie, nous tentons d'éclairer ces innovations locales grâce à une recherche empirique afin d'alimenter la réflexion sur ce qu'elles peuvent engendrer comme changement.

\section{Quelques pistes de réflexion sur base d'une recherche empirique}

Les initiatives locales de transition sont souvent considérées comme faisant partie des solutions pour construire une société alternative, comme l'a popularisé le film "Demain » (Cyril Dion et Mélanie Laurent, 2015). Or notre recherche tend à montrer que l'engagement des individus dans les différentes initiatives locales ne résulte pas nécessairement d'une volonté première de changer de système. Afin d'éclairer les motivations individuelles derrière les initiatives locales de transition, nous avons débuté il y a quelques mois une recherche de terrain $^{8}$ dans la ville de Gembloux sur le

${ }^{6}$ C. CASTORIADIS, Une société à la dérive. Entretiens et débats (1974-1997), Paris, Seuil, 2005, p. 244.

${ }^{7}$ S. LATOUCHE, op. cit., p. 251.

${ }^{8}$ Cette recherche est toujours en cours. Les premières observations, tirées d'une quinzaine d'entretiens avec les personnes porteuses de différentes initiatives de 
territoire de laquelle ont éclos plusieurs initiatives s'apparentant au mouvement de la transition. Toutes ne s'en réclament pas explicitement, mais elles ont en commun de promouvoir des alternatives au mode de vie capitaliste et consumériste. Les entretiens menés auprès des créateurs et/ou porteurs des différentes initiatives de transition permettent de mettre en évidence certains phénomènes relatifs à l'engagement. Dans le cadre de cette discussion, nous mobiliserons deux d'entre eux, à savoir l'importance du lien social et le rapport au politique entretenu par les différentes personnes interrogées.

D'abord, la majorité des personnes interviewées insistent sur l'importance de la dimension communautaire et conviviale des initiatives de transitions. En y participant, les individus cherchent à s'inscrire dans leur environnement direct en nouant des liens et en partageant des projets et expériences avec les gens qui vivent autour d'eux. La plupart des interviewés utilisent leur engagement pour créer un réseau social de proximité. Ils apprécient le fait de se sentir partie prenante d'un collectif local. Un facteur explicatif de cette motivation semble être lié au statut de nouvel habitant dans la commune. Ces personnes n'étant pas natives de la ville, il leur tient à cœur de développer de nouvelles attaches. Cet investissement sur le territoire via la construction de liens sociaux va de pair avec une défense de l'ancrage local (le village, le quartier). Certains précisent explicitement que l'important dans leur démarche était de développer un engagement social dans leur nouveau lieu de vie et que les objectifs liés à la transition étaient très secondaires dans leur implication. Si la transition en tant qu'objectif de changement social est un leitmotiv pour une bonne part des interviewés, elle ne semble pas être le moteur unique voire principal de l'engagement.

Le rapport au politique des acteurs engagés dans ces mouvements de la transition est extrêmement divers. Certains s'engagent d'abord et avant tout, dans un groupe de simplicité volontaire, par exemple, pour se changer eux-mêmes, pour donner sens à leur vie. Dans ces cas-là, l'objectif de changer la société n'est pas envisagé ou paraît hors d'atteinte. Les discours tenus par la majorité des interviewés font écho à un rejet généralisé du militantisme « traditionnel ${ }^{9}$, pour prôner un « engagement pratique » au

transition ont été présentées lors de la $5^{\text {è }}$ Conférence sur la Décroissance se déroulant à l'Université Corvinus de Budapest entre le 30 août et le 3 septembre 2016. Plus d'informations : http://budapest.degrowth.org/

9 L'utilisation d'instruments tels l'affiliation syndicale ou la participation à des manifestations ne fait pas partie de leur répertoire d'action. II s'agit moins de protester que d'agir pour une vie meilleure, à une échelle locale. 
niveau local. Selon Pleyers ${ }^{10}$, les citoyens s'engageant dans «la consommation critique et les mouvements conviviaux" refusent de se présenter comme étant des activistes, préférant expliquer leur attitude comme un changement dans leur propre mode de vie. En effet, les individus interrogés expliquent qu'en tant que citoyens actifs, ils veulent reprendre le contrôle sur différents éléments les affectant: alimentation, santé, vie en communauté, etc.

Parfois critiqué pour leur apolitisme et leur consensualisme mou ${ }^{11}$, nous percevons plutôt une diversité des rapports au politique parmi les militants de la transition. Certains veulent éviter à tout prix les contacts avec un système partisan qui, à leurs yeux, n'offre pas de véritables alternatives (et donc ils ne pourraient, ni ne voudraient participer à la gouvernance évoquée par Olivier De Schutter). Pour d'autres individus interrogés, les groupes liés à la transition portent en eux une dimension politique « au sens noble du terme ", dès l'instant où ils veulent créer des alternatives. Si la méfiance envers les partis traditionnels demeure très vivace pour la plupart des personnes interviewées, certains estiment que le politique peut être un partenaire ponctuel pour mener des projets.

\section{Conclusion}

Ces éléments empiriques offrent à notre sens une perspective complémentaire à ce que propose Olivier De Schutter pour envisager la sortie de l'imaginaire de croissance. II semble certain que le développement des initiatives locales a de beaux jours devant lui : le nombre d'initiatives et de personnes qui les rejoignent est en croissance. En revanche, il apparaît légitime de s'interroger sur leur capacité à influencer le système global et l'imaginaire social. A la suite de Geoffrey Pleyers, nous pouvons en effet nous demander : "Can the world be changed only by multiplying individual conversions or building local "resilient" communities? Will the multiplication of "alternative islands" in an ocean of modern capitalist societies manage to alter the system ? Or will actors also have to tackle more institutional and political struggles? ${ }^{12}$

\footnotetext{
${ }^{10}$ G. Pleyers, "The global Age : A social Movement Perspective " in Global modernity and social contestation, B. M. Bringen, J. M. Domingues (dir.), London, Sage, 2015.

11 J. Clotuche, E. Bouchat, «Transition : je t'aime moi non plus!», Politique, 92, novembre-décembre 2015, p. 33.

${ }^{12}$ G. PLEYERS, op. cit., p. 114.
} 